Title: Custom-made design of metabolite composition in $N$. benthamiana leaves using CRISPR activators.

\author{
Selma $S^{1}$, Sanmartín $N^{2}$, Espinosa-Ruiz $A^{1}$, Gianoglio $S^{1}$, Lopez-Gresa $M P^{1}$, Vázquez- \\ Vilar $\mathbf{M}^{1}$, Flors $\mathrm{V}^{2}$, Granell $\mathrm{A}^{1}$, Orzaez $\mathrm{D}^{1}$ \\ ${ }^{1}$ Instituto Biologia Molecular de Plantas, CSIC-UPV, Valencia, Spain \\ ${ }^{2}$ Escuela Superior de Tecnología y Ciencias Experimentales, Universidad Jaume I, Castellón de la Plana, \\ Spain.
}

Correspondence and requests for materials should be addressed to dorzaez@ibmcp.upv.es

\begin{abstract}
Transcriptional regulators based on CRISPR architecture expand our ability of reprogramming endogenous gene expression in plants. One of their potential applications is the customization of plant metabolome through the activation of selected enzymes in a given metabolic pathway. Using the previously described multiplexable CRISPR activator dCasEV2.1, we assayed the selective enrichment in Nicotiana benthamiana leaves of four different flavonoids, namely naringenin, eriodictyol, kaempferol and quercetin. After careful selection of target genes and guide RNAs combinations, we created successful activation programs for each of the four metabolites, each program activating between three and seven genes, and with individual gene activation levels ranging from 4- to 1500-fold. Metabolic analysis of the flavonoid profiles of each multigene activation program showed a sharp and selective enrichment of the intended metabolites and their glycosylated derivatives. Remarkably, principal component analysis of untargeted metabolic profiles clearly separated samples according to their activation treatment, and hierarchical clustering separated the samples in five groups, corresponding to the expected four highly enriched metabolite groups, plus an un-activated control. These results demonstrate that dCasEV2.1 is a powerful tool for re-routing metabolic fluxes towards the accumulation of metabolites of interest, opening the door for custom-made design of metabolic contents in plants.
\end{abstract}

Key Words: CRISPRa, Metabolic Engineering, Nicotiana benthamiana, Flavonoid Pathway.

\title{
Abbreviation list
}

4CL 4-coumaroyl CoA ligase

C4H Cinnamate 4-hydroxylase

$\mathrm{CHI}$ Chalcone isomerase

CHS Chalcone synthase 
DFR Dihydroflavonol 4-reductase

F3'H Flavonoid 3'-hydroxylase

F3H Flavanone 3-hydroxylase

FLS Flavonol synthase

PAL Phenylalanine ammonia-lyase

VPR VP64-P65-RtA

\section{Introduction}

Traditionally, plant breeding has contributed to the generation of crop varieties adapted to changing external conditions and to consumers' demands by selecting favourable genetic variants in a species' genomic pool, or by introducing new genetic traits through transgenesis or mutation by CRISPR (Jisha et al. 2015; Maioli et al. 2020). In recent years, the need for new adaptations has grown exponentially, fostered by climate change and human population dynamics, and the pressure to explore new breeding strategies has increased (Godwin et al. 2019). In this context, new breeding concepts inspired in Synthetic Biology propose the development of new programmable traits where physiological outputs (e.g., a developmental phase transition, or the activation of a metabolic pathway) occur as a response to endogenous or external inputs (e.g., a chemical cue, or an electromagnetic signal) perceived by synthetic sensors, and operated by engineered genetic operators (e.g., logic gates, toggle switches, oscillators, etc) (McKenzie et al. 1998; Ochoa-Fernandez et al. 2016; Bernabé-Orts et al. 2020). To produce the desired physiological output, operators need to be transcriptionally connected to a selected group of final actuators (e.g., a group of enzymes), that ultimately generate the designed phenotypic changes. Natural gene circuits have evolved intricated regulatory cascades of transcription factors (TFs) that connect operators with downstream actuators in a concerted manner, jointly generating a consistent physiological response. Among the many challenges facing Plant Synthetic Biology, a key one is to acquire the ability to customize the connections between synthetic operators and endogenous actuators in ways that are different to those designed by evolution but convenient for agriculture. Examples of new "synthetic" connections are the modification of flowering time (Papikian et al. 2019), the activation of an anticipated response to a forecasted biotic/abiotic stress (Chen et al. 2020), or the customization of metabolic composition (Llorente et al. 2020). Natural transcription factors, which are often used as connection hubs in traditional genetic engineering approaches (Xie et al. 2006; Naing et al. 2017), have a limited capacity for circuit customization due to their hardwired DNA binding specificities, which impede free selection of the downstream genes to be regulated. Recently, a new type of programmable transcriptional regulators (PTRs) based in CRISPR/Cas has emerged that allow easy customization of both DNA binding and transcriptional regulatory activities. The nuclease-inactivated CRISPR/Cas9 (dCas9) (Maeder et al. 2013) architecture 
enables the combination of autonomous activation domains (ADs) with the DNAbinding specificities of Cas9, which can be programmed through a 20 nucleotide-long guide RNA with minimum engineering efforts. In plants, several strategies to build potent these Programmable Transcriptional Activators (PTA) based in dCas9 have been described (Park et al. 2017; Lowder et al. 2018; Li et al. 2019; Pan et al. 2021), showing efficient activation of target endogenous genes. The two main advantages of dCas9PTA are (i) their high accuracy, reaching single-gene specificity levels as shown recently in transcriptomic analysis showing negligible off-target activation (Li et al. 2017); and (ii) their amenability for multiplexing (Vazquez-Vilar et al. 2016; Lowder et al. 2017). This latter feature enables the concerted activation of multiple actuators simultaneously, by simply loading the cell with several gRNAs, each one targeting a different promoter or a different position within a given promoter. The practical implications of multiplexing PTRs are widespread, from the design of synthetic regulatory cascades to the re-routing of endogenous metabolic fluxes. However, the capacities of PTRs in producing new-to-nature phenotypes are just starting to be explored, and no examples exist yet where PTRs are applied to re-route biosynthetic fluxes. Mastering the regulation of metabolic pathways would open the way for the customization of plant composition, a possibility with many implications in food and feed design, as well as in the development of plant biofactories.

The phenylpropanoid pathway is an alluring bioengineering target due to its pharmaceutical and industrial interest (Neelam et al. 2020). Besides, it is a highly branched pathway in plant secondary metabolism, offering interesting opportunities for biotechnological regulation and fit for the purpose of testing new technological approaches. The pathway can be divided into different parts (see Figure 1). The "general" section of the pathway generates cinnamic acid, coumaric acid and 4coumaroyl-CoA, the basic backbone products derive from phenylalanine, thus providing the core structures for the biosynthesis of all flavonoids as well as for other phenylpropanoid branches like the lignin pathway; a second group of reactions leads to the condensation and subsequent cyclization of the core structure, generating the first flavanones of the pathway; finally, flavanones will be the substrate for multiple reactions that originate the distinct subgroups of the flavonoid pathway, including flavonols, anthocyanins, isoflavonoids, etc (Nabavi et al. 2020). In plants, the main flavanones are naringenin, eriodictyol and hesperitin, which can also be found in their glycosylated forms and whose distribution changes between species and tissues. For example, in grapefruit and tomato naringenin glycosyl-conjugated compounds are the predominant flavanones present in the fruits, while in some species of citrus, like mandarin or lime, hesperitin glycosyl-conjugates are the most abundant flavanones (Khan et al. 2014). This variation is due to the different combination of gene expression patterns in the upstream part of the pathway. Similarly, the differential expression of downstream enzymes governs the predominant accumulation of flavonols (e.g. kaempferol, quercetin) or anthocyanins (e.g. delphinidin, pelargonidin), shaping important traits as fruit colour, antioxidant activity, etc. 
The flavonoid pathway has been the subject of remarkable metabolic engineering interventions mainly by making use of native transcription factors (Dias and Grotewold 2003; Park et al. 2021). The enzymes in the pathway are frequently regulated by a triad of TFs comprising a MYB TF, a bHLH and a WD repeat component (Zhao et al. 2013). In many cases, the overexpression of the MYB factor alone or in combination with a bHLH is sufficient to ectopically activate an entire branch of the pathway (Liu et al. 2015). MYB factors show certain degree of specificity for activating the biosynthesis of different flavonoid subgroups. Thus, whereas the ROSEA transcription factor activates enzymes in the anthocyanin branch in tomato and tobacco (Fresquet-Corrales et al. 2017; Vu and Lee 2019), the Arabidopsis thaliana MYB12 factor activates the enzymes of the flavonol subgroup, leading to the accumulation of kaempferol and rutin (quercetin glycosylate) as main products (Misra et al. 2010; Zhang et al. 2015). With the elucidation of the specificities of natural TFs from different species, followed by their recombinant expression, Butelli and co-workers obtained multi-level engineering of flavonoid compounds in tomato (Butelli et al. 2008). As shown in their work, the engineering precision obtained with native TFs reaches its limit at the "subgroup" level, as native TFs collectively activate those endogenous genes sharing similar regulatory sites in their 5'untranscribed regions, and these genes usually correspond to enzymes in the same branch of the pathway (e.g., flavanols, anthocyanins or flavanones branches). To achieve higher precision levels in plant metabolic engineering, up to the level of individual enzymes (and metabolites), endogenous TF seem not to be fit to the purpose and it would be necessary to break the evolutionary constrains and incorporate the type of single-gene specificity offered by PTAs.

In this work, we aimed to explore the efficiency and the precision limits of dCas9-PTAs for engineering the specialized metabolism in plants. Using the dCasEV2.1 programmable activator previously developed in our group (Selma et al. 2019), we first selected individual activation programs (i.e. single polycistronic constructs expressing of up to six gRNAs) for ten different target enzymes distributed in the general flavonoid pathway and the flavonone/ flavonol branch of the pathway. Then, we combined those enzymes in four groups, each group leading to the biosynthesis of a different flavonoid compound as final product. Four multigene activation programs (i.e., combinations of polycistronic gRNA constructs targeting several genes simultaneously) were constructed and assayed transiently in $N$. benthamiana, each program designed to specifically activate the genes in one of the four groups. As a result, four different and highly specific metabolic profiles were generated in the leaf, with a highly predominant accumulation of the expected target products in each of the assayed combinations. These results show that dCasEV2.1 raises metabolic engineering to a new precision level, opening the door for true customization of plant metabolic composition.

\section{Results}

Optimization of activation programs for individual genes in the flavonoid pathway. 
To engineer the flavonoid biosynthesis through the custom upregulation of endogenous genes, the first step consisted in the identification of flavonoid biosynthetic genes in Nicotiana benthamiana, including also those encoding upstream enzymes belonging to the general phenylpropanoid pathway. The KEGG reference database with the complete flavonoid pathway was used as a guide for the selection of all candidate genes (https://www.genome.jp/kegg/pathway.html). A schematic representation of the pathway can be found in Figure 1, where the enzymatic steps intended for transcriptional activation in this work are highlighted. Candidate gene identification in the $N$. benthamiana genome was carried out manually by homology search using orthologous proteins from $A$. thaliana, Solanum lycopersicum and Nicotiana tabacum available in Uniprot (https://www.uniprot.org/) and Solgenomics database (https://solgenomics.net/). The retrieved candidates were contrasted with the automatic annotation of the version v3.3 of the $N$. benthamiana genome assembly (https://www.nbenth.com/). The allotetraploid nature of $N$. benthamiana results in several paralogs and homoeologues for each enzymatic step in the pathway, therefore the selection of candidates for transcriptional activation was performed according to the following criteria: (i) maximum homology levels with the reference proteins; (ii) completeness of gene annotation, with reliable identification of the transcriptional start site (TSS), a critical parameter that determines the region in which activation efficiency is maximum, usually between nucleotides -100 to -300 from it. (iii) optimal sequence features for gRNA design in the activation region, with an absence of putative off-targets in the $N$. benthamiana genome. On some occasions, discrepancies between in silico gene annotation and transcriptomic information were found. In these cases, transcriptomic data was prioritized. In total, twelve different candidate genes were selected for upregulation in two optimization rounds (see below), covering eight enzymatic steps, namely PAL, CHS, CHI, C4H, 4CL, F3' $\mathrm{H}$ and F3H and FLS.

Once the candidate genes were selected, their transcriptional activation was assayed individually in $N$. benthamiana transient assays employing the dCasEV2.1 activation tool. The dCasEV2.1 comprises two modules, a constant module with two constitutively expressed transcriptional units (TUs), and a variable module carrying the gene-specific activation program. In the constant module, the first TU produces a deactivated Cas9 translationally fused to an EDLL activation domain (Tiwari et al. 2012), and the second TU expresses a VPR (Chavez et al. 2015) activation domain fused to the MS2 RNA aptamer-binding protein (Konermann et al. 2015) (Figure 2A). In each assay, the activation tool was completed with the co-transformation of the targetspecific module, which consists of one or two polycistronic gRNAs carrying three target-specific protospacers plus a MS2-binding RNA aptamer separated by processable tRNA spacers (Figure 2B). All protospacers were designed against the 5 ' untranscribed region of genes between -100 and $-300 \mathrm{bp}$ from the TSS. The list of the gRNAs target sites designed for each gene are listed in Table 1 and depicted in Figure 1. Four days post infiltration (dpi) the samples were collected, and levels of transcriptional activation were evaluated by RT-qPCR. The reference sample used as 
negative control was also infiltrated with dCasEV2.1 loaded with a non-target gRNA of $N$. benthamiana genome for a best comparison.

A first round of optimization was carried out with ten selected genes, namely NbPAL1, NbPAL2, NbC4H, NbCL4, NbCHS1, NbCHI2, NbF3H, NbF3'H, and NbFLS1. The NbDFR gene, previously assayed in our group was also added to the analysis for comparison. Most endogenous target genes showed remarkable upregulation upon dCasEV2.1 activation treatment (see Supplementary figure 1A). Surprisingly, the activation program designed for NbPAL1 resulted in a modest four-fold upregulation, while its homeologue NbPAL2 showed a 200-fold activation. This was a consequence of an erroneous identification of the TSS in NbPAL1, which generated the design of the gRNAs in sub-optimal positions. Consequently, NbPAL2 was selected in this work for further attempts to activate the phenylalanine ammonia-lyase. Unfortunately, the genes NbCHS1, NbCHI2 and NbFLS1 showed low activation rates in the first set of experiments. For this reason, a new optimization round for those suboptimal enzymatic steps was carried out by introducing new gRNAs design, or by targeting new alternative genes catalysing the same enzymatic step (Supplementary figure 1B). As presented in Figure $2 \mathrm{C}$, after the second optimization process, a successful transcriptional activation (> 10-fold) was obtained for all selected enzymatic steps except for $\mathrm{NbC4H}$ and $\mathrm{Nb4CL}$, where only modest four-fold activation rates were obtained. The best activation results were achieved for the previously described NbDFR (15000-fold) and NbCHS2 (18000-fold).

\section{Programming naringenin accumulation with dCasEV2.1}

The results obtained with the individual activation programs prompted us to undertake the simultaneous activation of several genes in the pathway following a modular polycistronic gRNA strategy (Lowder et al. 2017; Hashimoto et al. 2018). As a first step, we aimed at the co-activation of the enzymatic steps leading to the accumulation of naringenin, therefore involving the genes NbPAL2, NbC4H, Nb4CL, NbCHS2, NbCHI1 and $\mathrm{NbCHI2}$. To select the best dCasEV2.1 activation program possible, we assayed seven different gRNA multiplexing arrangements targeting all the above-mentioned genes except $\mathrm{NbC4H}$. Each combination comprised between three to six U6-26-driven polycistronic TUs (Figure $3 \mathrm{~A}$ ). $\mathrm{NbC4H}$ was omitted as a target gene in all gRNA combinations due to its modest activation rates. All seven combined activation programs were transiently assayed in $N$. benthamiana leaves following the same methodology described for previous experiments. The RT-qPCR results in Figure 3B show that all the assayed programs resulted in significant gene activation, although induction levels were notably reduced compared with programs addressing individual genes. In general, it was observed that smaller multiplexing arrays resulted in higher activation rates of individual genes. For instance, NbCHS2 activation reached between 800- and 1200-fold with activation programs AP-N1, AP-N1B, AP-N2 and AP-N3 comprising 2 or 3 target genes but dropped below 600 -fold in programs targeting four or five genes (AP-N2B and AP-N4B). Following the same trend, those targets showing 
modest activations in single-gene programs showed even lower inductions with complex activation programs, dropping below significance levels in some cases such as $\mathrm{NbCHI2}$ when treated with AP-N2B or AP-N4B.

In order to see if the changes in transcript profiles have resulted in the expected metabolic changes, naringenin levels in treated samples were analysed by LC-MS (UPLC-(ESI)-QTOFMS) at 4 and $7 \mathrm{dpi}$ using a purified commercial standard for identification (Figure 3C). As shown in Figure 3D, naringenin content was enriched in all samples as compared with a control leaf where dCasEV2.1 was loaded with an unrelated program. Maximum levels of naringenin were obtained with AP-N3, which targeted NbPAL2, NbCHS2 and NbCHI1 simultaneously. In this combination, the levels of the target compound were raised almost 100-fold as compared with the levels in control samples. The upregulation of NbPAL seemed important for the early activation of the pathway, since the best levels of naringenin accumulation were found in samples where this enzyme was upregulated. The accumulation trends of the targeted metabolite were similar at 4 and $7 \mathrm{dpi}$, although a drop in signal intensity was observed in the later time point, particularly in samples AP-N3, AP-N4 and AP-N4B.

\section{Customization of flavonoid composition as a study case for single metabolite precision level engineering.}

Despite the indications that complex gRNA programs resulted in lower activation efficiencies than simpler ones, the remarkable levels of naringenin accumulation obtained with shorter programs suggested that there was still room for adding new instructions to AP-N3, thus extending regulation further downstream in the pathway. Furthermore, naringenin constitutes a crossroad point from which several compounds can be derived depending on the set of downstream enzymes to be activated. Thus, the activation of $\mathrm{F3}^{\prime} \mathrm{H}$ on top of AP-N3 would convert naringenin into a different flavanone, eriodictyol. Moreover, naringenin can be used as starting point for the accumulation of two important flavonols, kaempferol and quercetin. Steering the metabolic flux to produce kaempferol would require the simultaneous activation of two enzymes, first $F 3 H$ to produce dihydrokaempferol, and next $F L S$ to introduce a double bound bond in the $\mathrm{C}$ ring, yielding kaempferol. Alternatively, the production of quercetin can be induced by taking the eriodictyol program as a basis, and adding activation instructions for $F 3 H$ and $F L S$, producing first dihydroquercetin and finally quercetin. Following this rationale, three new metabolite-specific gRNA programs (Figure 4A), namely AP-K, AP-E and AP-Q, were constructed and assayed in $N$. benthamiana next to AP-N3 to produce kaempferol, eriodictyol, quercetin and naringenin, respectively. Figure $4 \mathrm{~B}$ shows the gene activation profiles obtained for each combination, where it can be observed that an increase in program complexity has a negative effect on the overall induction levels compared to simpler programs. This was clearly observed in the activation levels of NbCHS2, which drops by approximately 5 -fold when more than four genes are targeted simultaneously. Despite the progressive reduction in activation levels, a significant upregulation is still observed 
in all enzymes assayed even in the more complex program (AP-K and AP-Q). It is worth noticing that $\mathrm{NbF}^{\prime} \mathrm{H}$ is only activated in AP-Q but not in AP-K, this serving as an additional indication of the specificity of the activation programs and discarding positive feedback as cause for the observed upregulations.

To understand to what extent the customized activation of different subsections of the pathway led to differential flavonoid composition in treated leaves, an untargeted LCMS metabolomic analysis was carried out with the same samples previously analysed by RT-qPCR. As the Principal Component Analysis (PCA) in Figure 5A indicates, each activation program produced a distinct and characteristic metabolite profile, with a first main component separating controls from flavonoid-activated samples accounting for $28.9 \%$ of the variance, and a second principal component separating flavonones Activation Programs to the flavonols Activation Programs accounting for $23.4 \%$ of the variance. The level of precision achieved with the four activation programs became even more evident when the 100 most significantly different features were hierarchically clustered (Figure 5B). Here, a perfect clustering was observed that parallels the activation programs and the control sample. A detailed version of the heatmap can be found in Supplementary figure 3. The differential $m / z$ ions and their respective retention times are listed in Supplementary table 2. Furthermore, when metabolites in each cluster were tentatively identified attending to their retention time and the characteristic $\mathrm{m} / \mathrm{z}$ ratios (see Materials and Methods), a remarkable match was found between the activation program employed and the predominant metabolites in the samples. Thus, as expected, AP-N3 samples accumulate naringenin aglycon and three other glycoside derivatives more than any other sample. Eriodictyol and its sugar conjugates are the metabolites accounting for the aggrupation of the APE hierarchical cluster, although certain levels of eriodictyol are also found in the AP-N3 cluster. This is not surprising since both flavanones are only one enzymatic step away from each other, and eriodictyol can be also synthetized from coumaric acid following a secondary branch in the pathway (see Figure 1). Remarkably, flavonols are almost completely absent both in AP-N3 and AP-E. On the contrary, kaempferol and its glycosylated derivatives are the main differentially accumulated compounds in AP-K samples, and conversely, the quercetin aglycon and its conjugated derivatives are most abundant in AP-Q samples. As could be anticipated, a certain level of crosscontamination is observed in both flavonol programs. Again, this is not entirely unexpected as both AP-K and AP-Q share 5 out of 6 steps in their respective programs. It is worth noting that flavonol levels remain low in AP-K and AP-Q samples, indicating that successful activation of downstream genes is responsible for the specific accumulation of flavonol compounds.

\section{Discussion}

Programmable transcriptional activators (PTAs) based on CRISPR/dCas9 architecture are powerful tools for the transcriptional regulation of a wide spectrum of targets. After a first generation of PTAs based on the direct translational fusion of ADs to dCas 9 
showed modest induction activities (Piatek et al. 2015), subsequent generations have emerged incorporating multiple $A D$ anchoring sites such as multi-epitope chains (Papikian et al. 2019), RNA aptamers or combinations of them. These upgraded versions considerably boosted PTAs ability to produce strong activation of targeted genes (Lowder et al. 2018; Lee et al. 2019). These improvements, added to the multiplexing capacity of CRISPR/dCas9, have turned CRISPR-PTAs into extraordinary tools for Plant Synthetic Biology applications. Of particular interest is the ability offered by CRISPR-PTAs to tinkering with enzyme expression in metabolic pathways, thus shaping the plant metabolite composition. In this work we tinkered with a section of the flavonoid pathway, exploring the limits of the tool, to find out that dCasEV2.1, the CRISPR-PTA developed in our laboratory, offers an unprecedented level of precision in plant metabolic engineering interventions. We anticipate that other CRISPR-PTAs developed by other groups, having an equivalent mode of action and showing similar gene activation levels, would perform similarly well when used for the same purposes. We show that with a careful selection of gRNAs, simultaneous regulation of up to six enzymatic steps can be achieved, leading to the highly preferential accumulation of individual flavanones (naringenin or eriodictyol) and flavonols (quercetin or kaempferol). The ability to harness a pathway towards the production of a single predominant metabolite has important implications in plant biomanufacturing and biorefinery, since the isolation of pure valuable metabolites is known to be hindered by the presence of contaminant compounds belonging to the same pathway, hence showing similar chemical properties. In the examples described here, a considerable proportion of the targeted metabolites were found in glycosylated forms. $N$. benthamiana, as many other plant species, is promiscuous in glycosyl transferase activities (Wang 2009). From a biomanufacturing point of view, glycosylated forms constitute a relatively minor problem due to the affordability of industrial glycosylases. As an alternative, glycosyl transferases could be also targeted for programmable repression, leading to the predominant accumulation of the aglycon.

In this work we only use transcriptional activators as tools to re-route metabolic fluxes. Programmable repression is partially dispensable for the control of the flavonoid pathway because in $N$. benthamiana leaves the flux through the pathway is low. However, the interplay of programmable repressors would enable to further refine the accuracy of re-routing programs. As mentioned above, the repression of glycosyl transferases would serve to ensure the predominance of aglycon forms if required. Furthermore, as shown in Figure 1, eriodictyol can be synthetized by an alternative route that involves the action of $\mathrm{NbC3}^{\prime} \mathrm{H}, \mathrm{NbC3H}$ and $\mathrm{NbHCT}$ genes. Programmed repression of one or more of these genes could contribute to reduce the levels of accompanying metabolites when the production of a single flavonol is attempted. Furthermore, programmable repression will be strongly required for harnessing metabolic pathways other than flavonoid pathway which are highly active by default. Unfortunately, there are few examples in the literature showing a highly efficient repression based on CRISPR architecture (Tang et al. 2017). All currently described tools, although helpful, are probably insufficient in providing full control due to their 
inability to conduct strong transcriptional repressions in highly active genes. It has been proposed that dCas9, due to its mode of action when binding DNA, which implies a relaxation of the chromatin in the surrounding area, has limited capacity to act as a strong repressor when fused to conventional repressor domains. This limitation might be circumvented by the use of epigenetic repressors adding reversible chromatin silencing marks (Gallego-Bartolomé 2020). Alternatively, programmable repression could be achieved by other means, such as post-transcriptional gene silencing tools (Mahas et al. 2019). Given the remarkable ability of dCasEV2.1 to program transcriptional activation, its combination with efficient repressors would enable near full control of metabolic pathways in plants.

For the delivery of activation programs, we made use of transient Agrobacteriumbased transformation. Transient delivery of genetic information in the form of T-DNA or RNA is becoming increasingly popular in plant biotechnology. Recently, transient reprogramming of crop plants was shown using RNA virus-based delivery systems, either as viral particles or mediated by Agrobacterium (Torti et al. 2021). Agroinfiltration has become not only a widely used experimental procedure (Norkunas et al. 2018; Grosse-Holz et al. 2018), but also a potent and scalable plant biomanufacturing strategy as recently demonstrated with the production of plantmade vaccines against influenza and SARS-CoV2 (D'Aoust et al. 2008; Diego-Martin et al. 2020). On the other hand, the gRNA elements in PTAs can be also transiently delivered using viral vectors, as recently shown using TRV as delivery agent (Ghoshal et al. 2020). Reprogramming metabolic pathways using transient tools has the additional advantage of circumventing the need for stable transgenics, giving regulatory advantages in some areas. Alternatively, stable transformation of the activation programs could also be envisioned as a powerful tool in crop breeding. Stable integration in the plant's genome could circumvent the complexity limits that have been evidenced in this work, which could be attributed to the cargo capacity of the TDNAs or its ability to cope with highly repetitive gRNA structures. Stable transgenic programs could be pyramided in different genomic loci, avoiding limits imposed by TDNA cargo capacity. If required by the presence of many gRNA-coded instructions, the expression of the remaining components in dCasEV2.1 could be boosted with state-ofthe-art strategies, preventing them from becoming a limiting factor for activation (Pasin et al. 2017). Connecting integrated programs with endogenous or exogenous cues using appropriated genetic sensors (Ochoa-Fernandez et al. 2020; Randall 2021) would provide the ultimate ability to customize plant metabolic composition using its own endogenous metabolic pathways.

In sum, we show here that CRISPR/dCas9-based transcriptional activators provide the sufficient precision and multiplexing capacity to reprogram metabolic pathways and customize metabolic composition in plants. This ability has important implications in feed and food design, as well as in the valorisation of industrial crops.

\section{Methods}




\section{DNA constructs}

All plasmids used in this work were assembled using GoldenBraid (GB) cloning (SarrionPerdigones et al. 2013). The DNA sequences of the constructs generated in this work are available at https://gbcloning.upv.es/ by entering the IDs provided in Supplementary table 3. Briefly, the multiplexing vectors used for this work were generated as GB level -1 vectors and previously described in Selma et al. (Selma et al. 2019). The level -1 vectors, pVD1_M1-3pTRNA scf 2.1 (GB1436) pVD1_M2-3pTRNA scf 2.1 (GB1437) and pVD1_M3-3pTRNA scf 2.1 (GB1438) were used to assemble the protospacer sequences occupying the first, second and third positions in the final gRNA assembly. For GB gRNA assemblies two partially complementary primers containing the protospacer sequence designed at https://gbcloning.upv.es/do/crispr/multi cas9 gRNA domesticator 1 by entering the protospacer sequences listed in Supplementary table 1. Primers were resuspended in water to final concentrations of $1 \mu \mathrm{M}$ and equal volumes of forward and reverse primer were mixed. The mixture was incubated at room temperature for $5 \mathrm{~min}$ for the hybridization of the primer pair. $1 \mu \mathrm{l}$ of the primer mix was included in a BsmBI restriction-ligation reaction with $75 \mathrm{ng}$ of pUPD2 and $75 \mathrm{ng}$ of the corresponding level -1 vector for the assembly of tRNA-protospacer-scaffold units in level 0 . The next step consists in the assembly of the multiplexing gRNA expression cassette in level 1 . For level 1 assemblies, $75 \mathrm{ng}$ of level 0 gRNA for each position, $75 \mathrm{ng}$ of U626 promoter (GB1001), and $75 \mathrm{ng}$ of pDGB3 $\alpha$ destination vector were included in a Bsal restrictionligation reaction.

The combination of level 1 multiplexing gRNAs and dCasEV2.1 TUs were performed by binary Bsal or $\mathrm{BsmBI}$ restriction-ligation reactions to obtain all the level $\geq 1$ assemblies as it was described in Sarrion-Perdigones, A. et al. (Sarrion-Perdigones et al. 2013)

\section{Nicotiana benthamiana agroinfiltration}

The transient expression assays were carried out through agroinfiltration of $N$. benthamiana leaves. $N$. benthamiana plants were grown for 5 weeks before agroinfiltration in a growing chamber where the growing conditions were $24{ }^{\circ} \mathrm{C} / 20^{\circ} \mathrm{C}$ light/darkness with a $16 \mathrm{~h} / 8 \mathrm{~h}$ photoperiod. The plasmids were transferred to $A$. tumefaciens strain GV3101 by electroporation. Agroinfiltration was carried out with overnight grown bacterial cultures. The cultures were pelleted and resuspended in agroinfiltration solution (10 mM MES, pH 5.6, $10 \mathrm{mM} \mathrm{MgCl}$, and $200 \mu \mathrm{M}$ acetosyringone). After incubation for $2 \mathrm{~h}$ at room temperature with agitation, the optical density of the bacterial cultures was adjusted to 0.1 at $600 \mathrm{~nm}$ and mixed for co-infiltration with the silencing suppressor P19. Agroinfiltrations were carried out through the abaxial surface of the three youngest fully expanded leaves of each plant with a $1 \mathrm{ml}$ needle-free syringe. 


\section{RNA isolation and RT-qPCR Gene Expression Analysis}

Total RNA was isolated from $100 \mathrm{mg}$ agroinfiltrated leaves using the Gene Jet Plant Purification Mini Kit (Thermo Fisher Scientific) according to the manufacturer's instructions. The timing for sample collection was 4 days post infiltration (dpi) for testing individual genes activation and for naringenin optimization and $5 \mathrm{dpi}$ in the case of AP-N3, AP- E, AP-K, AP-Q activation. Before CDNA synthesis, total RNA was treated with the rDNAse-I Invitrogen Kit according to the manufacturer instructions. An aliquot of $1 \mu \mathrm{g}$ of DNAse treated RNA was used for cDNA synthesis using the PrimeScript $^{\mathrm{TM}}$ RT-PCR Kit (Takara) in a final volume of $20 \mu \mathrm{L}$ according to the manufacturer indications. Expression levels for each gene were measured in triplicated reactions, in the presence of a fluorescent dye (SYBR ${ }^{\circledR}$ Premix Ex Taq) using Applied biosystem 7500 Fast Real Time PCR system with specific primer pairs (Supplementary table 4). The F-box gene was used as internal reference (Liu et al. 2012). Basal expression levels were calculated with samples agroinfiltrated with dCasEV2.1 in combination with an unspecific gRNA. mRNA fold change calculations for each sample were carried out according the comparative $\Delta \Delta C T$ method (Livak and Schmittgen 2001).

Liquid chromatography (LC) and ESI mass spectrometry (MS) for flavonoids content analysis

Leaf samples of three different plants agroinfiltrated with each construct were collected at 4 and 5 dpi respectively and used as triplicates for metabolomics analyses. The same tissue was used for transcriptomics and metabolomics analyses. The tissue was frozen in liquid $\mathrm{N}_{2}$ and powdered with a grind mill and, finally, lyophilized. Thirty milligrams of dried tissue were extracted at $4^{\circ} \mathrm{C}$ with $1 \mathrm{~mL}$ of methanol $30 \%$ containing $0.01 \%$ of formic acid. The preparation was homogenized with a grind mill and kept in ice during $20 \mathrm{~min}$. After that, the samples were centrifugated at $15000 \mathrm{rpm}$ for 15 minutes. The supernatant was collected and filtrated with 20 microns cellulose strainer (Regenerated Cellulose Filter, Teknokroma). Three independent biological and two technical replicates per sample were analysed. $20 \mu \mathrm{l}$ of each sample were injected into an Acquity UPLC system (Waters) coupled to a hybrid quadrupole time-of-flight instrument (QTOF MS Premier). Separation was performed using an HPLC SunFire C18 analytical column with a particle size of $5 \mu \mathrm{m}, 2.1 \times 100 \mathrm{~mm}$ (Waters). A gradient of methanol and water containing $0.01 \%$ formic acid was used. The gradient started with $95 \%$ aqueous solvent and a flow of $0.3 \mathrm{ml}$ per minute. The gradient reached $50 \%$ of aqueous solvent at $8 \mathrm{~min}$, increasing the level of organic solvent to $95 \%$ at 12 minutes. The gradient was kept in isocratic conditions for $1 \mathrm{~min}$ and later returned to initial conditions in 2 minutes. The column could equilibrate for 3 minutes, for a total of 22 minutes per sample.

Data were collected in MS and MS/MS mode to gain structural information of the detected metabolites. The MS/MS function was programmed in a range of 5 to $45 \mathrm{eV}$ 
t-wave to obtain each analyte fragmentation spectrum (Pastor et al. 2018). The electrospray ionization was performed in positive and negative mode and analysed individually in order to obtain a best profile of the flavonoid metabolites following the specifications described by Gamir et al. (Gamir et al. 2012).

For unequivocal metabolite determination, samples of naringenin and eriodictyol chemical standard (Sigma) were analysed in the same conditions. The exact mass, specific retention time, and spectrum fragmentation of naringenin and eriodyctiol standard were compared to the fragmentation profiles of each sample as described by Schymanski et al. (Schymanski et al. 2014).

\section{Naringenin content data analysis and statistics}

The raw data obtained were processed by Masslynx 4.1 software and transformed to .cdf files. The positive (ESI+) and negative (ESI-) signals were analysed separately. Naringenin identification was carried out by comparison with a purified standard also analysed in the same conditions.

The quasimolecular ion with $\mathrm{m} / z 271.06$ in ESI negative mode, retention time (5.47 min.) and the fragmentarion ions $\mathrm{m} / \mathrm{z} 151$ and $\mathrm{m} / \mathrm{z} 119$ allowed to unequivocally identify naringenin in the samples (Supplementary figure 2).

Metabolite amounts were quantified based on the normalized peak area units relative to their respective dry weight. All statistical analyses were conducted with Statgraphics Centurion software for the ANOVA statistical analysis $(p<0.05)$ and means were expressed with the standard error.

\section{Untargeted data analysis and statistics}

The raw data obtained were processed and transformed to .cdf files. The negative (ESI) signals were analysed employing the xcms software (https://xcmsonline.scripps.edu/) for comparing all the samples. TIC normalization was applied to each biological triplicate with a baseline of 20 peak intensity relative units. All peaks with a signal lower than 300 in all samples were eliminated to reduce the background. The data analysed comprises the retention time between 1 to $6.5 \mathrm{~min}$ (corresponding to the elution conditions for phenolic compounds) and $\mathrm{m} / \mathrm{z}$ values ranging 200 to 1000 . Finally, a cut-off of $75 \%$ in the coefficient of variation was applied between biological triplicates.

The data obtained was analysed using the MetaboAnalyst5.0 Software (https://www.metaboanalyst.ca/). Logarithmic transformation and Pareto scaling were employed as normalization to elaborate the Principal Component Analysis and the Hierarchical Clustering and Heatmap. Euclidean distance and Ward Clustering algorithm were applied as parameters for elaborating Hierarchical Clustering, and an ANOVA test was the statistical method used for generated the $100 \mathrm{~m} / \mathrm{z}$ more significantly different in each group. 
The $m / z$ values obtained as significantly different in each group were manually clustered into single metabolites employing the original chromatograms. Finally, the tentative identification of each metabolite was carried out using external databases (https://pubchem.ncbi.nlm.nih.gov/) and the information obtained with their fragmentation profiles and collected in Supplementary table 2. The quantification of the metabolites was performed employing the parental ion identified in the F1.

\section{Author contributions}

D.O. and S.S. designed the experiments. S.S. and N.S, conducted the experiments. AR.A. and L-G.M contributes with data analysis. D.O. and S.S drafted the manuscript. G.S V-V.M. F.V and G.T contribute with the manuscript writing and editing. All the authors discussed and revised the manuscript.

\section{Acknowledgements}

This work has been funded by Grant BIO2016-78601-R and PID2019-108203RB-10 Plan Nacional I+D, Spanish Ministry of Economy and Competitiveness and Spanish Ministry of Science and Innovation. Sara Selma is a recipient of FPI fellowship associated with this Grant (BES-2017-080098).

\section{Conflict of interest}

The authors declare no conflicts of interest.

\section{References}

Bernabé-Orts JM, Quijano-Rubio A, Vazquez-Vilar M, Mancheño-Bonillo J, Moles-Casas V, Selma S, Gianoglio S, Granell A, Orzaez D (2020) A memory switch for plant synthetic biology based on the phage $\varphi C 31$ integration system. Nucleic Acids Res 48(6):33793394. https://doi.org/10.1093/nar/gkaa104

Butelli E, Titta L, Giorgio M, Mock H-P, Matros A, Peterek S, Schijlen EGWM, Hall RD, Bovy AG, Luo J, Martin C (2008) Enrichment of tomato fruit with health-promoting anthocyanins by expression of select transcription factors. Nature Biotechnology 26(11):1301-1308. https://doi.org/10.1038/nbt.1506

Chavez A, Scheiman J, Vora S, Pruitt BW, Tuttle M, P R Iyer E, Lin S, Kiani S, Guzman CD, Wiegand DJ, Ter-Ovanesyan D, Braff JL, Davidsohn N, Housden BE, Perrimon N, Weiss R, Aach J, Collins JJ, Church GM (2015) Highly efficient Cas9-mediated transcriptional programming. Nat Methods 12(4):326-328. https://doi.org/10.1038/nmeth.3312

Chen J, Clinton M, Qi G, Wang D, Liu F, Fu ZQ (2020) Reprogramming and remodeling: transcriptional and epigenetic regulation of salicylic acid-mediated plant defense. Journal of Experimental Botany 71(17):5256-5268. https://doi.org/10.1093/jxb/eraa072 
D’Aoust M-A, Lavoie P-O, Couture MM-J, Trépanier S, Guay J-M, Dargis M, Mongrand S, Landry N, Ward BJ, Vézina L-P (2008) Influenza virus-like particles produced by transient expression in Nicotiana benthamiana induce a protective immune response against a lethal viral challenge in mice. Plant Biotechnology Journal 6(9):930-940. https://doi.org/10.1111/j.1467-7652.2008.00384.x

Dias AP, Grotewold E (2003) Manipulating the accumulation of phenolics in maize cultured cells using transcription factors. Biochemical Engineering Journal 14(3):207-216. https://doi.org/10.1016/S1369-703X(02)00225-5

Diego-Martin B, González B, Vazquez-Vilar M, Selma S, Mateos-Fernández R, Gianoglio S, Fernández-del-Carmen A, Orzáez D (2020) Pilot Production of SARS-CoV-2 Related Proteins in Plants: A Proof of Concept for Rapid Repurposing of Indoor Farms Into Biomanufacturing Facilities. Frontiers in Plant Science 11:2101. https://doi.org/10.3389/fpls.2020.612781

Fresquet-Corrales S, Roque E, Sarrión-Perdigones A, Rochina M, López-Gresa MP, Díaz-Mula HM, Bellés JM, Tomás-Barberán F, Beltrán JP, Cañas LA (2017) Metabolic engineering to simultaneously activate anthocyanin and proanthocyanidin biosynthetic pathways in Nicotiana spp. PLOS ONE 12(9):e0184839. https://doi.org/10.1371/journal.pone.0184839

Gallego-Bartolomé J (2020) DNA methylation in plants: mechanisms and tools for targeted manipulation. New Phytologist 227(1):38-44. https://doi.org/10.1111/nph.16529

Gamir J, Pastor V, Cerezo M, Flors V (2012) Identification of indole-3-carboxylic acid as mediator of priming against Plectosphaerella cucumerina. Plant physiology and biochemistry : PPB 61:169-179. https://doi.org/10.1016/j.plaphy.2012.10.004

Ghoshal B, Vong B, Picard CL, Feng S, Tam JM, Jacobsen SE (2020) A viral guide RNA delivery system for CRISPR-based transcriptional activation and heritable targeted DNA demethylation in Arabidopsis thaliana. PLoS Genet 16(12):e1008983-e1008983. https://doi.org/10.1371/journal.pgen.1008983

Godwin ID, Rutkoski J, Varshney RK, Hickey LT (2019) Technological perspectives for plant breeding. Theoretical and Applied Genetics 132(3):555-557. https://doi.org/10.1007/s00122-019-03321-4

Grosse-Holz F, Madeira L, Zahid MA, Songer M, Kourelis J, Fesenko M, Ninck S, Kaschani F, Kaiser M, van der Hoorn RAL (2018) Three unrelated protease inhibitors enhance accumulation of pharmaceutical recombinant proteins in Nicotiana benthamiana. Plant Biotechnology Journal 16(10):1797-1810. https://doi.org/10.1111/pbi.12916

Hashimoto R, Ueta R, Abe C, Osakabe Y, Osakabe K (2018) Efficient Multiplex Genome Editing Induces Precise, and Self-Ligated Type Mutations in Tomato Plants. Frontiers in Plant Science 9:916. https://doi.org/10.3389/fpls.2018.00916

Jisha V, Dampanaboina L, Vadassery J, Mithöfer A, Kappara S, Ramanan R (2015) Overexpression of an AP2/ERF Type Transcription Factor OsEREBP1 Confers Biotic and Abiotic Stress Tolerance in Rice. PLOS ONE 10(6):e0127831. https://doi.org/10.1371/journal.pone.0127831 
Khan MK, Zill-E-Huma, Dangles O (2014) A comprehensive review on flavanones, the major citrus polyphenols. Journal of Food Composition and Analysis 33(1):85-104. https://doi.org/10.1016/j.jfca.2013.11.004

Konermann S, Brigham MD, Trevino AE, Joung J, Abudayyeh OO, Barcena C, Hsu PD, Habib N, Gootenberg JS, Nishimasu H, Nureki O, Zhang F (2015) Genome-scale transcriptional activation by an engineered CRISPR-Cas9 complex. Nature 517(7536):583-588. https://doi.org/10.1038/nature14136

Lee JE, Neumann M, Duro DI, Schmid M (2019) CRISPR-based tools for targeted transcriptional and epigenetic regulation in plants. PLOS ONE 14(9):e0222778. https://doi.org/10.1371/journal.pone.0222778

Li Z, Wang F, Li J-F (2019) Targeted Transcriptional Activation in Plants Using a Potent Dead Cas9-Derived Synthetic Gene Activator. Current protocols in molecular biology 127(1):e89-e89. https://doi.org/10.1002/cpmb.89

Li Z, Zhang D, Xiong X, Yan B, Xie W, Sheen J, Li J-F (2017) A potent Cas9-derived gene activator for plant and mammalian cells. Nature Plants 3(12):930-936. https://doi.org/10.1038/s41477-017-0046-0

Liu D, Shi L, Han C, Yu J, Li D, Zhang Y (2012) Validation of Reference Genes for Gene Expression Studies in Virus-Infected Nicotiana benthamiana Using Quantitative Real-Time PCR. PLOS ONE 7(9):e46451. https://doi.org/10.1371/journal.pone.0046451

Liu J, Osbourn A, Ma P (2015) MYB Transcription Factors as Regulators of Phenylpropanoid Metabolism in Plants. Molecular Plant 8(5):689-708. https://doi.org/10.1016/j.molp.2015.03.012

Livak KJ, Schmittgen TD (2001) Analysis of Relative Gene Expression Data Using Real-Time Quantitative PCR and the 2- $\Delta \triangle$ CT Method. Methods 25(4):402-408. https://doi.org/10.1006/meth.2001.1262

Llorente B, Torres-Montilla S, Morelli L, Florez-Sarasa I, Matus JT, Ezquerro M, D'Andrea L, Houhou F, Majer E, Picó B, Cebolla J, Troncoso A, Fernie AR, Daròs J-A, RodriguezConcepcion M (2020) Synthetic conversion of leaf chloroplasts into carotenoid-rich plastids reveals mechanistic basis of natural chromoplast development. Proceedings of the National Academy of Sciences 117(35):21796 LP - 21803. https://doi.org/10.1073/pnas.2004405117

Lowder LG, Paul JW, Qi Y (2017) Multiplexed Transcriptional Activation or Repression in Plants Using CRISPR-dCas9-Based Systems. In: Kaufmann K, Mueller-Roeber B (eds) Plant Gene Regulatory Networks: Methods and Protocols. Springer New York, New York, NY, pp 167-184

Lowder LG, Zhou J, Zhang Y, Malzahn A, Zhong Z, Hsieh T-F, Voytas DF, Zhang Y, Qi Y (2018) Robust Transcriptional Activation in Plants Using Multiplexed CRISPR-Act2.0 and mTALE-Act Systems. Molecular Plant 11(2):245-256. https://doi.org/10.1016/j.molp.2017.11.010

Maeder ML, Linder SJ, Cascio VM, Fu Y, Ho QH, Joung JK (2013) CRISPR RNA-guided activation of endogenous human genes. Nat Methods 10(10):977-979. https://doi.org/10.1038/nmeth.2598 
Mahas A, Aman R, Mahfouz M (2019) CRISPR-Cas13d mediates robust RNA virus interference in plants. Genome Biol 20(1):263-263. https://doi.org/10.1186/s13059-019-1881-2

Maioli A, Gianoglio S, Moglia A, Acquadro A, Valentino D, Milani AM, Prohens J, Orzaez D, Granell A, Lanteri S, Comino C (2020) Simultaneous CRISPR/Cas9 Editing of Three PPO Genes Reduces Fruit Flesh Browning in Solanum melongena L. Frontiers in Plant Science 11:1883. https://doi.org/10.3389/fpls.2020.607161

McKenzie MJ, Mett VV, PH SR, Jameson PE (1998) Controlled cytokinin production in transgenic tobacco using a copper-inducible promoter. Plant physiology 116(3):969977. https://doi.org/10.1104/pp.116.3.969

Misra P, Pandey A, Tiwari M, Chandrashekar K, Sidhu OP, Asif MH, Chakrabarty D, Singh PK, Trivedi PK, Nath P, Tuli R (2010) Modulation of transcriptome and metabolome of tobacco by Arabidopsis transcription factor, AtMYB12, leads to insect resistance. Plant Physiol 152(4):2258-2268. https://doi.org/10.1104/pp.109.150979

Nabavi SM, Šamec D, Tomczyk M, Milella L, Russo D, Habtemariam S, Suntar I, Rastrelli L, Daglia M, Xiao J, Giampieri F, Battino M, Sobarzo-Sanchez E, Nabavi SF, Yousefi B, Jeandet $P$, Xu S, Shirooie S (2020) Flavonoid biosynthetic pathways in plants: Versatile targets for metabolic engineering. Biotechnology Advances 38:107316-107316. https://doi.org/10.1016/j.biotechadv.2018.11.005

Naing AH, Park KI, Ai TN, Chung MY, Han JS, Kang Y-W, Lim KB, Kim CK (2017) Overexpression of snapdragon Delila (Del) gene in tobacco enhances anthocyanin accumulation and abiotic stress tolerance. BMC Plant Biol 17(1):65-65. https://doi.org/10.1186/s12870017-1015-5

Neelam, Khatkar A, Sharma KK (2020) Phenylpropanoids and its derivatives: biological activities and its role in food, pharmaceutical and cosmetic industries. null 60(16):2655-2675. https://doi.org/10.1080/10408398.2019.1653822

Norkunas K, Harding R, Dale J, Dugdale B (2018) Improving agroinfiltration-based transient gene expression in Nicotiana benthamiana. Plant Methods 14(1):71. https://doi.org/10.1186/s13007-018-0343-2

Ochoa-Fernandez R, Abel NB, Wieland F-G, Schlegel J, Koch L-A, Miller JB, Engesser R, Giuriani G, Brandl SM, Timmer J, Weber W, Ott T, Simon R, Zurbriggen MD (2020) Optogenetic control of gene expression in plants in the presence of ambient white light. Nature Methods 17(7):717-725. https://doi.org/10.1038/s41592-020-0868-y

Ochoa-Fernandez R, Samodelov SL, Brandl SM, Wehinger E, Müller K, Weber W, Zurbriggen MD (2016) Optogenetics in Plants: Red/Far-Red Light Control of Gene Expression. Methods in molecular biology (Clifton, NJ) 1408:125-139. https://doi.org/10.1007/978-1-4939-3512-3_9

Pan C, Wu X, Markel K, Malzahn AA, Kundagrami N, Sretenovic S, Zhang Y, Cheng Y, Shih PM, Qi $Y$ (2021) CRISPR-Act3.0 for highly efficient multiplexed gene activation in plants. Nature Plants. https://doi.org/10.1038/s41477-021-00953-7

Papikian A, Liu W, Gallego-Bartolomé J, Jacobsen SE (2019) Site-specific manipulation of Arabidopsis loci using CRISPR-Cas9 SunTag systems. Nature communications 10(1):729. https://doi.org/10.1038/s41467-019-08736-7 
Park CH, Xu H, Yeo HJ, Park YE, Hwang G-S, Park NI, Park SU (2021) Enhancement of the flavone contents of Scutellaria baicalensis hairy roots via metabolic engineering using maize Lc and Arabidopsis PAP1 transcription factors. Metabolic Engineering 64:64-73. https://doi.org/10.1016/j.ymben.2021.01.003

Park J-J, Dempewolf E, Zhang W, Wang Z-Y (2017) RNA-guided transcriptional activation via CRISPR/dCas9 mimics overexpression phenotypes in Arabidopsis. PLOS ONE 12(6):e0179410. https://doi.org/10.1371/journal.pone.0179410

Pasin F, Bedoya LC, Bernabé-Orts JM, Gallo A, Simón-Mateo C, Orzaez D, García JA (2017) Multiple T-DNA Delivery to Plants Using Novel Mini Binary Vectors with Compatible Replication Origins. ACS Synth Biol 6(10):1962-1968. https://doi.org/10.1021/acssynbio.6b00354

Pastor V, Sánchez-Bel P, Gamir J, Pozo MJ, Flors V (2018) Accurate and easy method for systemin quantification and examining metabolic changes under different endogenous levels. Plant Methods 14(1):33-33. https://doi.org/10.1186/s13007-018-0301-z

Piatek A, Ali Z, Baazim H, Li L, Abulfaraj A, Al-Shareef S, Aouida M, Mahfouz MM (2015) RNAguided transcriptional regulation in planta via synthetic dCas9-based transcription factors. Plant Biotechnology Journal 13(4):578-589. https://doi.org/10.1111/pbi.12284

Randall RS (2021) The plant AlcR-pAlcA ethanol-inducible system displays gross growth artefacts independently of downstream pAlcA-regulated inducible constructs. Scientific Reports 11(1):2142. https://doi.org/10.1038/s41598-020-80903-z

Sarrion-Perdigones A, Vazquez-Vilar M, Palaci J, Castelijns B, Forment J, Ziarsolo P, Blanca J, Granell A, Orzaez D (2013) GoldenBraid 2.0: A Comprehensive DNA Assembly Framework for Plant Synthetic Biology. PLANT PHYSIOLOGY 162(3):1618-1631. https://doi.org/10.1104/pp.113.217661

Schymanski EL, Gerlich M, Ruttkies C, Neumann S (2014) Solving CASMI 2013 with MetFrag, MetFusion and MOLGEN-MS/MS. Mass spectrometry (Tokyo, Japan) 3(Spec Iss 2):S0036-S0036. https://doi.org/10.5702/massspectrometry.S0036

Selma S, Bernabé-Orts JM, Vazquez-Vilar M, Diego-Martin B, Ajenjo M, Garcia-Carpintero V, Granell A, Orzaez D (2019) Strong gene activation in plants with genome-wide specificity using a new orthogonal CRISPR/Cas9-based programmable transcriptional activator. Plant Biotechnol J 17(9):1703-1705. https://doi.org/10.1111/pbi.13138

Tang X, Levi G. Lowder, Zhang T, Malzahn AA, Zheng X, Voytas DF, Zhong Z, Chen Y, Ren Q, Li Q, Kirkland ER, Zhang Y, Qi Y (2017) A CRISPR-Cpf1 system for efficient genome editing and transcriptional repression in plants. Nature Plants 3(February):1-5. https://doi.org/10.1038/nplants.2017.18

Tiwari SB, Belachew A, Ma SF, Young M, Ade J, Shen Y, Marion CM, Holtan HE, Bailey A, Stone JK, Edwards L, Wallace AD, Canales RD, Adam L, Ratcliffe OJ, Repetti PP (2012) The EDLL motif: a potent plant transcriptional activation domain from AP2/ERF transcription factors: Strong plant transcriptional activation domain. The Plant Journal 70(5):855-865. https://doi.org/10.1111/j.1365-313X.2012.04935.x 
Torti S, Schlesier R, Thümmler A, Bartels D, Römer P, Koch B, Werner S, Panwar V, Kanyuka K, Wirén N von, Jones JDG, Hause G, Giritch A, Gleba Y (2021) Transient reprogramming of crop plants for agronomic performance. Nat Plants 7(2):159-171.

https://doi.org/10.1038/s41477-021-00851-y

Vazquez-Vilar M, Bernabé-Orts JM, Fernandez-del-Carmen A, Ziarsolo P, Blanca J, Granell A, Orzaez D (2016) A modular toolbox for gRNA-Cas9 genome engineering in plants based on the GoldenBraid standard. Plant Methods 12(1):10.

https://doi.org/10.1186/s13007-016-0101-2

Vu AT, Lee JM (2019) Genetic variations underlying anthocyanin accumulation in tomato fruits. Euphytica 215(12):196. https://doi.org/10.1007/s10681-019-2519-x

Wang X (2009) Structure, mechanism and engineering of plant natural product glycosyltransferases. FEBS Letters 583(20):3303-3309.

https://doi.org/10.1016/j.febslet.2009.09.042

Xie D-Y, Sharma SB, Wright E, Wang Z-Y, Dixon RA (2006) Metabolic engineering of proanthocyanidins through co-expression of anthocyanidin reductase and the PAP1 MYB transcription factor. The Plant Journal 45(6):895-907. https://doi.org/10.1111/j.1365-313X.2006.02655.x

Zhang Y, Butelli E, Alseekh S, Tohge T, Rallapalli G, Luo J, Kawar PG, Hill L, Santino A, Fernie AR, Martin C (2015) Multi-level engineering facilitates the production of phenylpropanoid compounds in tomato. Nature Communications 6:1-11. https://doi.org/10.1038/ncomms9635

Zhao L, Gao L, Wang H, Chen X, Wang Y, Yang H, Wei C, Wan X, Xia T (2013) The R2R3-MYB, bHLH, WD40, and related transcription factors in flavonoid biosynthesis. Functional \& Integrative Genomics 13(1):75-98. https://doi.org/10.1007/s10142-012-0301-4 


\section{Figures Captions:}

Figure 1: Schematic representation of the flavonoid biosynthetic pathway in plants. The different metabolites are represented in boxes. The genes that integrate the pathway are: ANS (anthocyanidin synthase), CHS (Chalcone synthase), CHI (Chalcone isomerase), CCOAOMT (Caffeoyl-CoA O-methyltransferase), $\mathrm{C3H}$ (4-coumarate 3-hydroxylase), C3'H (4-coumaroyl shikimate/quinate 3'-hydroxylase), $\mathrm{C} 4 \mathrm{H}$ (Cinnamate 4-hydroxylase), 4CL (4-coumaroyl CoA ligase), DFR (Dihydroflavonol 4-reductase), F3H (Flavanone 3-hydroxylase), F3'H (flavonoid 3'hydroxylase), F3'-5H (Flavonoid 3' 5-hydroxylase), F3'-5'H (Flavonoid 3' 5'-hydroxylase), FLS (Flavonol synthase), HCT (4-hydroxycinnamoyl CoA:shikimate/quinate hydroxycinnamoyltransferase), PAL (Phenylalanine ammonia-lyase). The genes marked in red are those selected for transcriptional activation with dCasEV2.1 in this work. A representation of each candidate gene promoter with the gRNA positions of the first round of optimization in red and the second in blue is included. The asterisks represent the gRNA position recalculated with updated information of the TSS available in databases (https://www.nbenth.com/).

Figure 2: dCasEV2.1-mediated transcriptional activation of individual genes of the flavonoid pathway in $\mathbf{N}$. benthamiana. A) Schematic representation of the dCasEV2.1 activator, comprising the dCas9 fused to the EDLL activation domain in C-terminus and the coat protein of the phage MS2 fused to the activation domain VPR. B) Schematic representation of the polycistronic gRNA2.1 array including tRNA-spaced gRNAs with MS2-binding RNA aptamers in the $3^{\prime}$ end of the scaffold. C) mRNA fold change at 4 dpi obtained by targeting the endogenous genes with optimized gRNAs in N. benthamiana leaves. The gR1 or gR2 indicate the round of optimization where the gRNAs were selected. The NbCHS2 gR2ab activation was performed with 2 set of gRNAs. The NbDFR gR1 is included as internal control of activation. Bars represent average fold change $+/-$ SD $(n=3)$. Asterisks indicate T student significant values ( $\left.{ }^{*}=p<0.05\right)$. Images were created with BioRender.com.

Figure 3: Optimization of naringenin production in $N$. benthamiana leaves through dCasEV2.1 activation. A) Representation of the different activation programs (AP)s tested for activating of naringenin production (AP-N1, AP-N1B, AP-N2, AP-N2B, AP-N3, AP-N4 and APN4B). B) mRNA fold change at 4 dpi obtained by targeting the endogenous genes of the flavonoid pathway with optimized gRNAs in $N$. benthamiana leaves. Bars represent average fold change +/- SD $(n=3)$. C) Identification of naringenin in $N$. benthamiana leaves by comparison with a true naringenin commercial standard (STD naringenin) using the ion with $\mathrm{m} / \mathrm{z} 271.06$ in ESI negative mode. C-sample: negative control, AP-N sample, dCasEV2.1activated sample. D) Relative naringenin quantification in the indicated samples at 4- and 7dpi. Bars represent average intensity. Different letters indicate statistically significant differences (ANOVA, Tukey HSD test; $p<0.01, n=3$ ). Asterisks indicate T student significant values $(*=p<0.05)$. 
Figure 4: Design and relative expression analyses of activation programs (APs) for naringenin, eriodictyol, kaempferol and quercetin production in $N$. benthamiana. A) Representation of the Activation Programs (APs), the gRNAs and the targeted genes included in each of them (AP-N3 for Naringenin Activation Program, AP-E for Eriodictyol Activation Program, AP-K for Kaempferol Activation Program and AP-Q for Quercetin Activation Program). B) mRNA fold change at 5 dpi obtained by targeting the endogenous genes with optimized gRNAs in N. benthamiana leaves for the groups AP-N3, AP-E, AP-K and AP-Q. Bars represent average fold change $+/$ - SD $(n=3)$. Asterisks indicate $T$ student significant values $\left(^{*}=\right.$ $\mathrm{p}<0.05)$

Figure 5: Analysis of the metabolic profiles of $\boldsymbol{N}$. benthamiana leaves treated with activation programs (APs) for naringenin, eriodictyol, kaempferol and quercetin. A) Principal Component Analysis resulting from the untargeted LC-MS data obtained in ESI negative mode from leaves treated with programs AP-N3, AP-E, AP-K and AP-Q and control samples (C-) agroinfiltrated with unprogrammed dCasEV2.1. B) Hierarchical cluster analysis and heatmap representation of the Control, AP-N3, AP-E, AP-K and AP-Q metabolic profiles, with three biological replicates per condition (ESI-). The metabolites represented are the 100 most significant using a t-test analysis $(p<0.05)$ of each sample. The data was obtained using Euclidean distance and Ward's minimum variance method. Red indicates up-regulated and blue downregulated features. Each $\mathrm{m} / \mathrm{z}$ corresponds to the same compound in all samples. C) Schematic representation of selected target metabolites of the flavonoid pathway and the genes involved in each enzymatic reaction required for their biosynthesis. The coloured bars represent the targeted gene for each group (AP-N3 in orange, AP-E in green, AP-K in blue and $A P-Q$ in red). D) Relative abundance of identified metabolites. The ions employed for the metabolic quantification are the parental ions identified in the heatmap as single compounds (See also Supplementary table 2). N, Naringenin; N-H, Naringenin Hexose; N-G. Naringenin Glucoside; E, Eriodictyol; E-H, Eriodictyol Hexose; E-G, Eriodictyol Glucoside; K-DH, Kaempferol Dihexose; K-DHDO, Kaempferol Dihexose-deoxyhexose; K-H, Kaempferol Hexose; K-G, Kaempferol Glucoside; Q-DH, Quercetin Dihexose; Q-DHDO, Dihexose-deoxyhexose; Q-HDO, Quercetin Hexose-deoxyhexose; Q-H, Quercetin Hexose; Q-G, Quercetin Glucoside. 


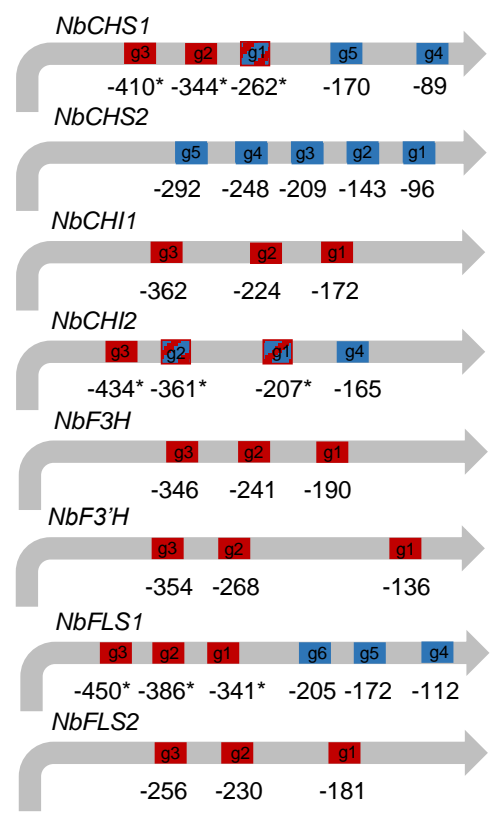

NbCHS1

$\mathrm{NbCHS}$
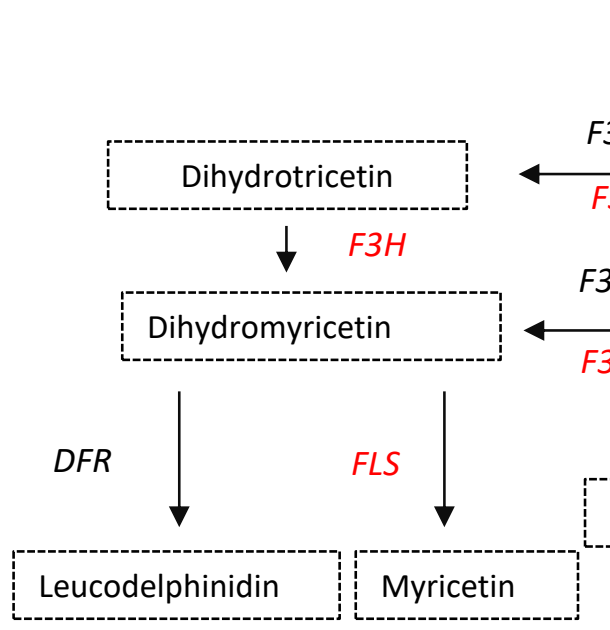

\section{ANS}

Delphinidin

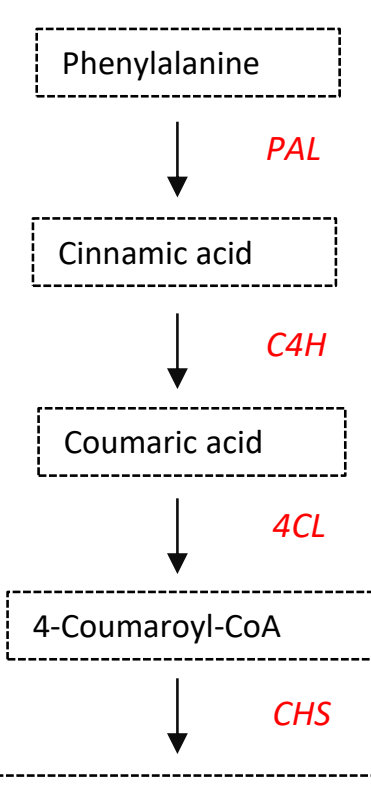

Naringerin-Chalcone
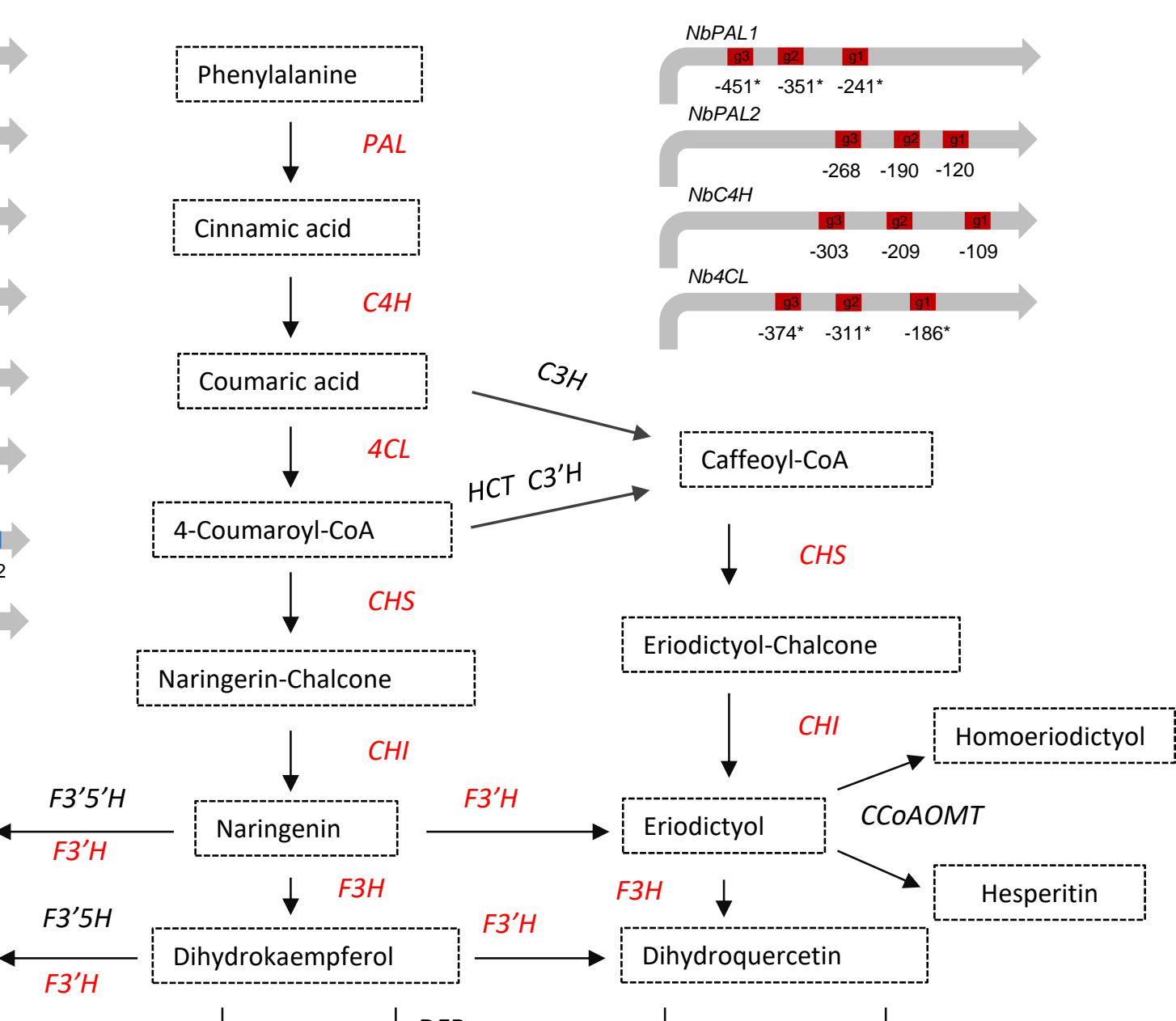

Kaempferol

Leucopelargonidin

ANS

Pelargonidin

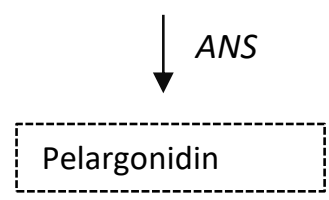

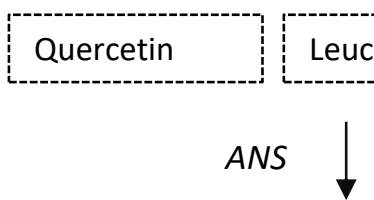

Cyanidin 
A

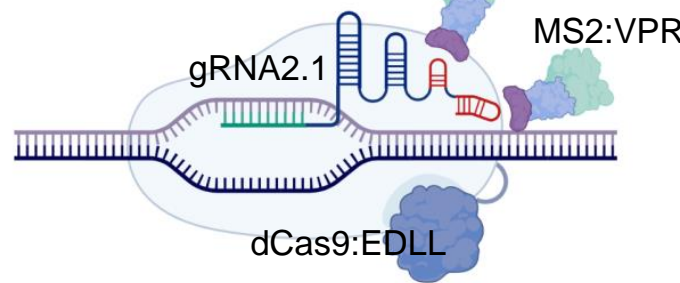

B

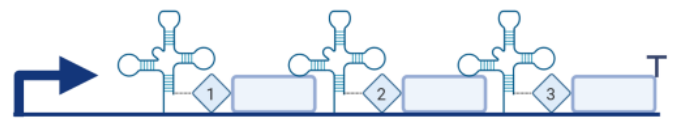

Polycistronic gRNA2.1

C
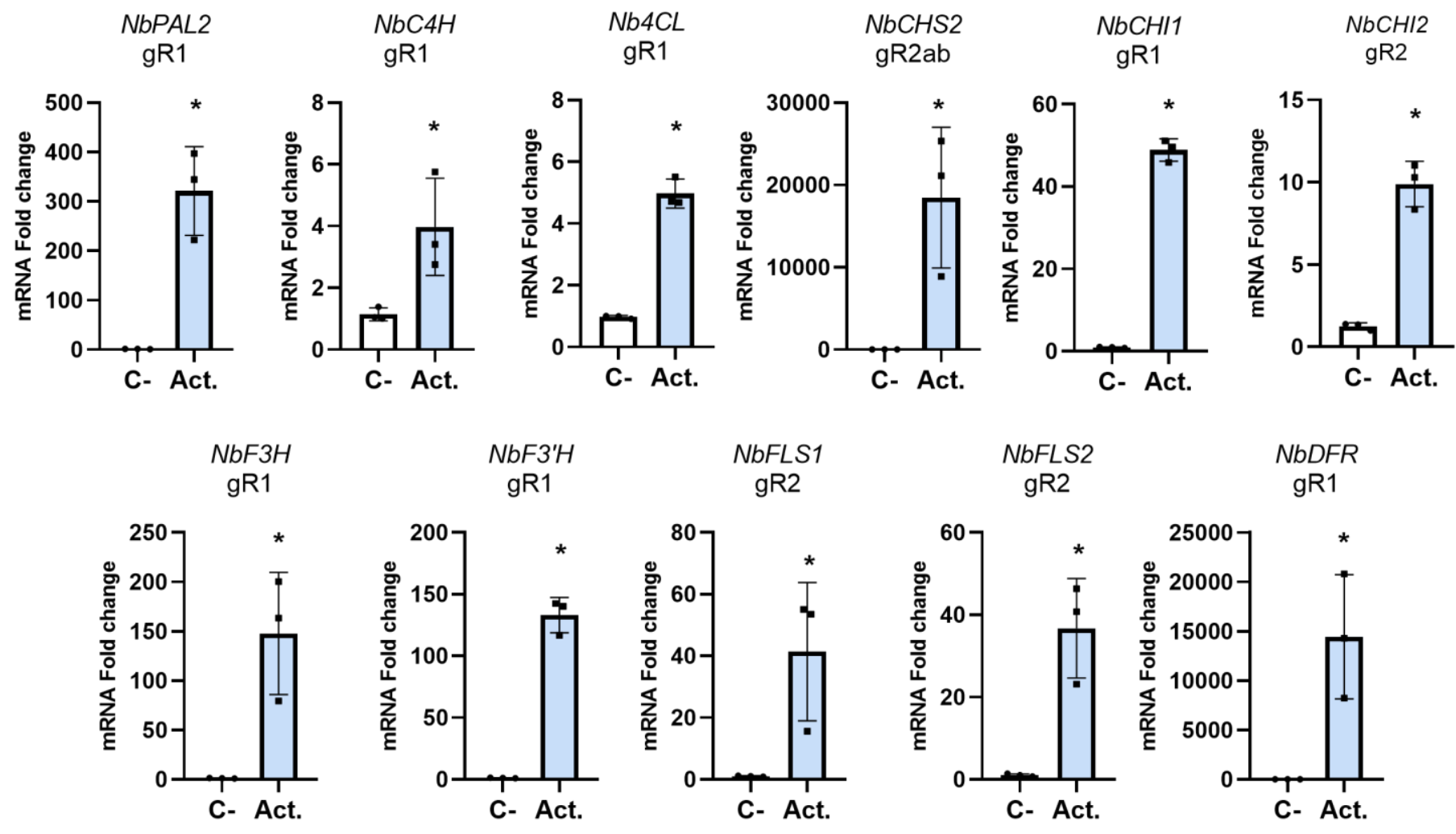
A

bioRxiv preprint doi: https://doi.org/10.1101/2021.07.12.452005; this version posted July 12, 2021. The copyright holder for this preprint (which was not certified by peer review) is the author/funder. All rights reserved. No reuse allowed without permission.

NbPAL2 gR1

NbCHS2 gR2ab

$\mathrm{NbCHI1} g R 1$

$\mathrm{NbCHI2} g R 2$

$N b 4 C L g R 1$

AP-N1

AP-N1B

AP-N2

AP-N2B

AP-N3

AP-N4

AP-N4B

\section{$3 x$}

$3 x$
$3 x$

B
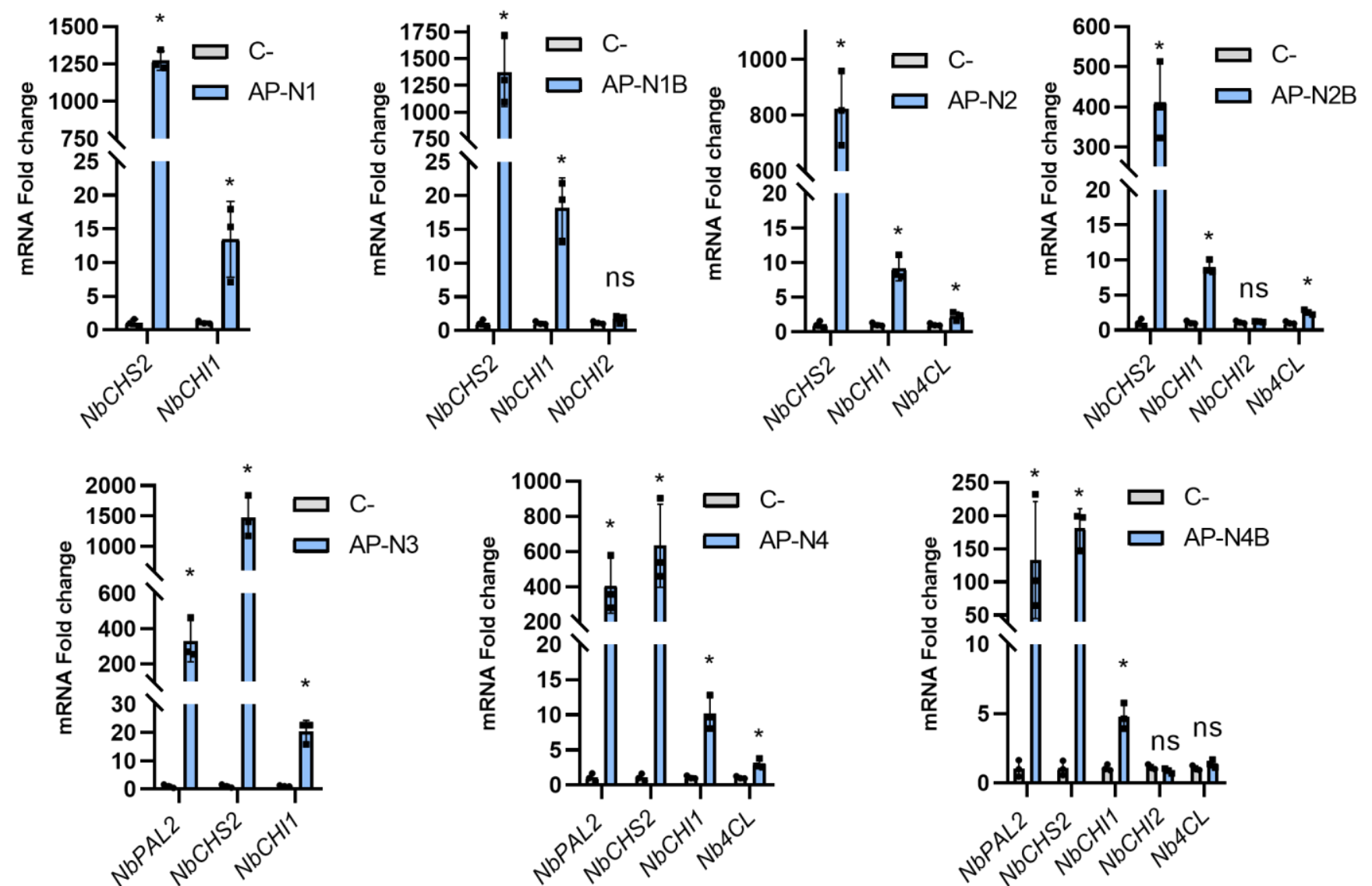

C

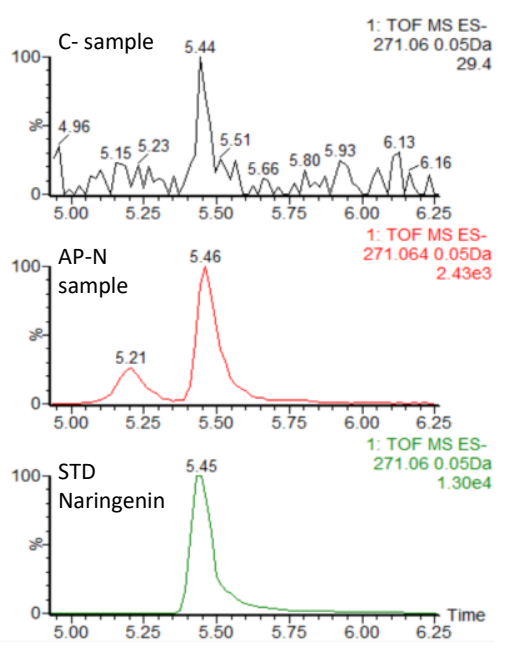

D
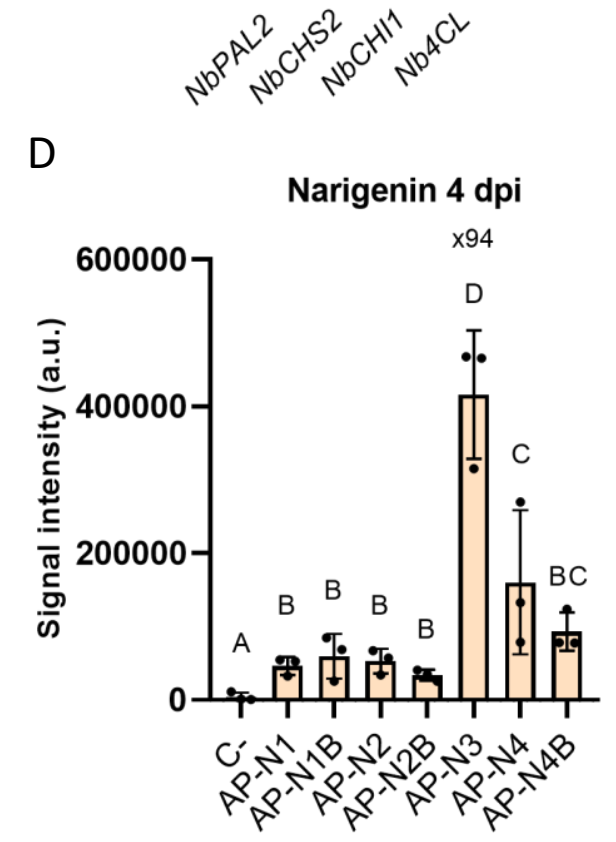

Narigenin 4 dpi
$3 x$

$3 x$

$3 x$

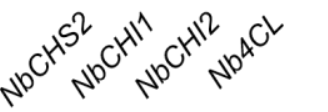

Narigenin 7 dpi

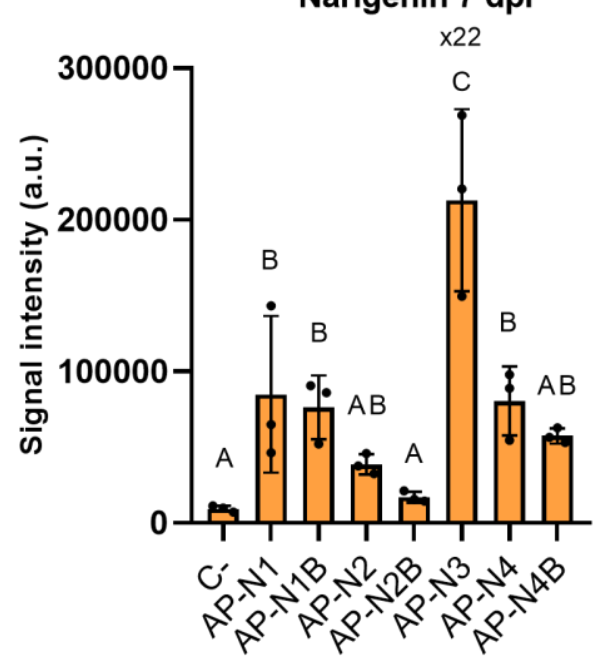


bioRxiv preprint doi: https://doi.org/10.1101/2021.07.12.452005; this version posted July 12, 2021. The copyright holder for this preprint A (which was not certified by peer review) is the author/funder. All rights reserved. No reuse allowed without permission.

\begin{tabular}{|c|c|c|c|c|c|c|c|}
\hline & $\begin{array}{c}\text { NbPAL2 } \\
g R 1\end{array}$ & $\begin{array}{c}\text { NbCHS2 } \\
\text { gR2ab }\end{array}$ & $\begin{array}{c}\mathrm{NbCHI1} \\
\mathrm{gR1}\end{array}$ & $\begin{array}{c}\text { NbF3'H } \\
g R 1 \\
\end{array}$ & $\begin{array}{c}N b F 3 H \\
g R 1 \\
\end{array}$ & $\begin{array}{c}\text { NbFLS1 } \\
g R 2 \\
\end{array}$ & $\begin{array}{c}\text { NbFLS2 } \\
g R 2 \\
\end{array}$ \\
\hline AP-N3 & $3 x$ & $5 x$ & $3 x$ & & & & \\
\hline AP-E & $3 x$ & $5 x$ & $3 x$ & $3 x$ & & & \\
\hline AP-K & $3 x$ & $5 x$ & $3 x$ & & $3 x$ & $3 x$ & $3 x$ \\
\hline$A P-Q$ & $3 x$ & $5 x$ & $3 x$ & $3 x$ & $3 x$ & $3 x$ & $3 x$ \\
\hline
\end{tabular}

B
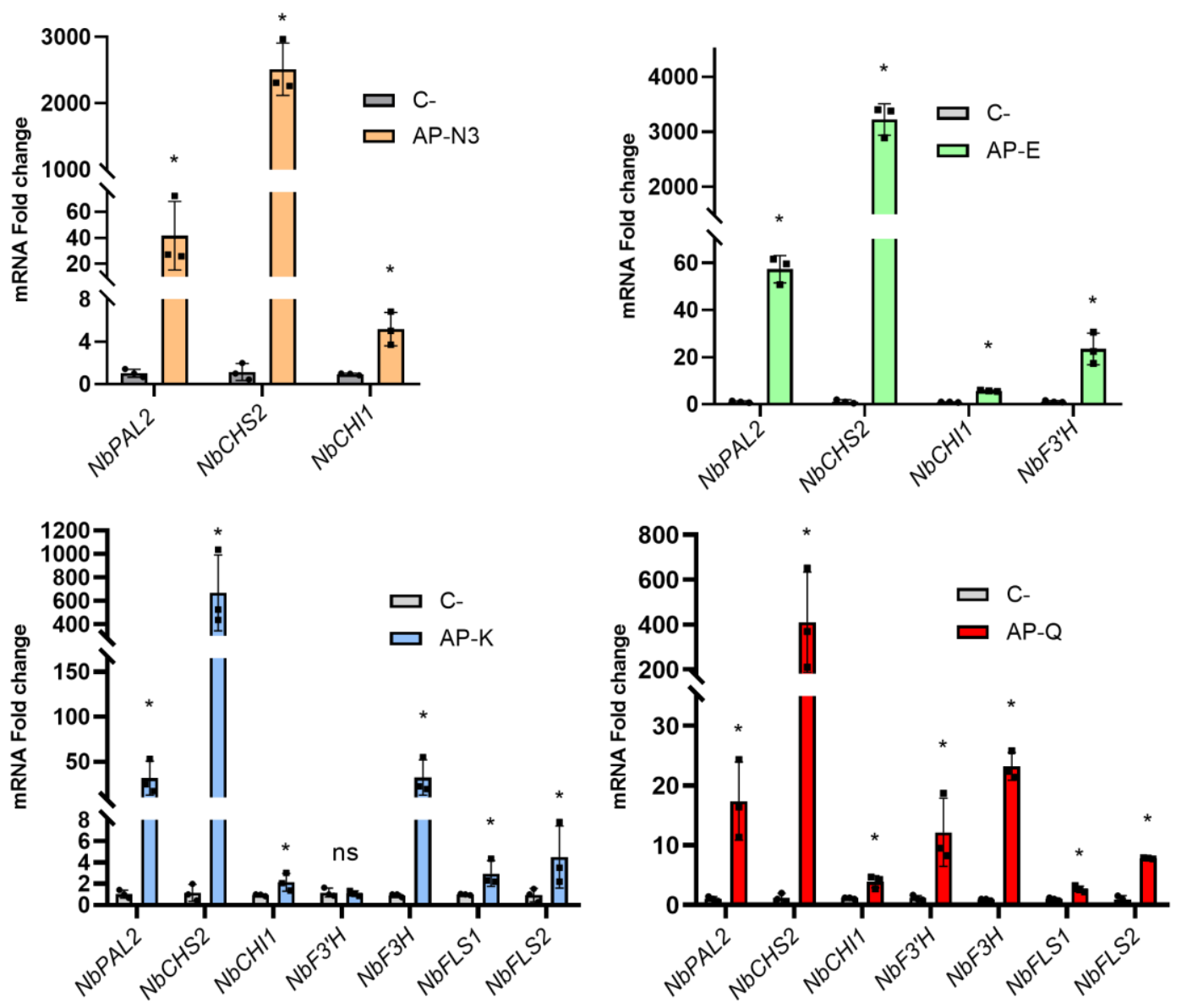
A

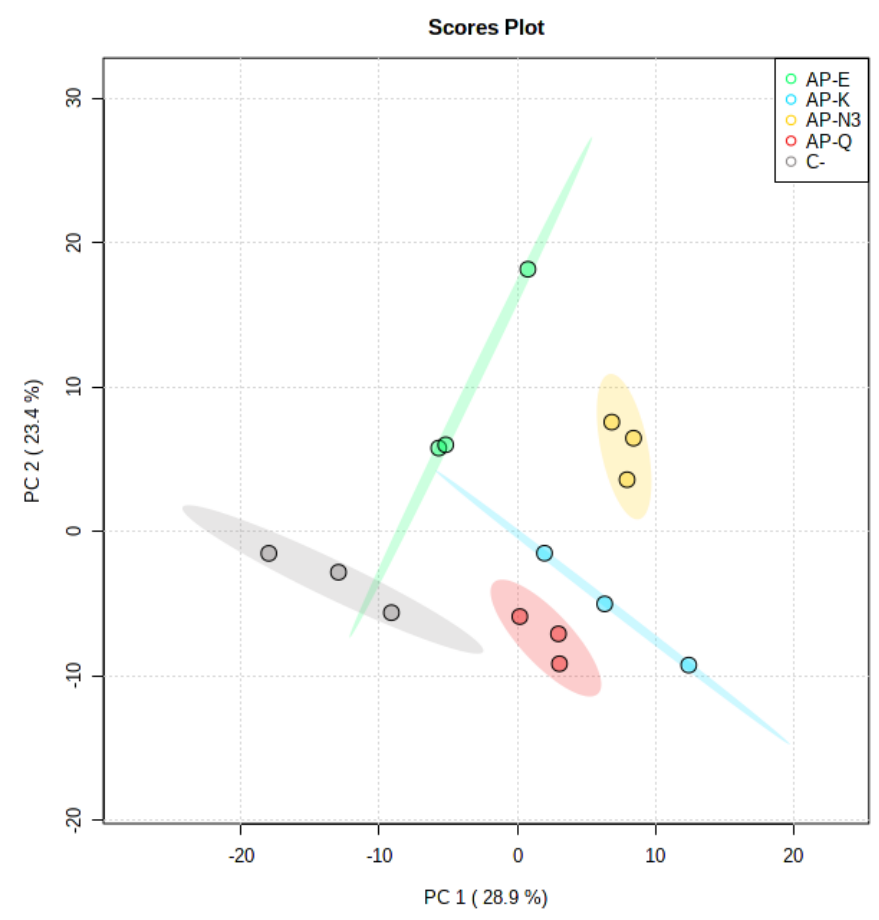

C

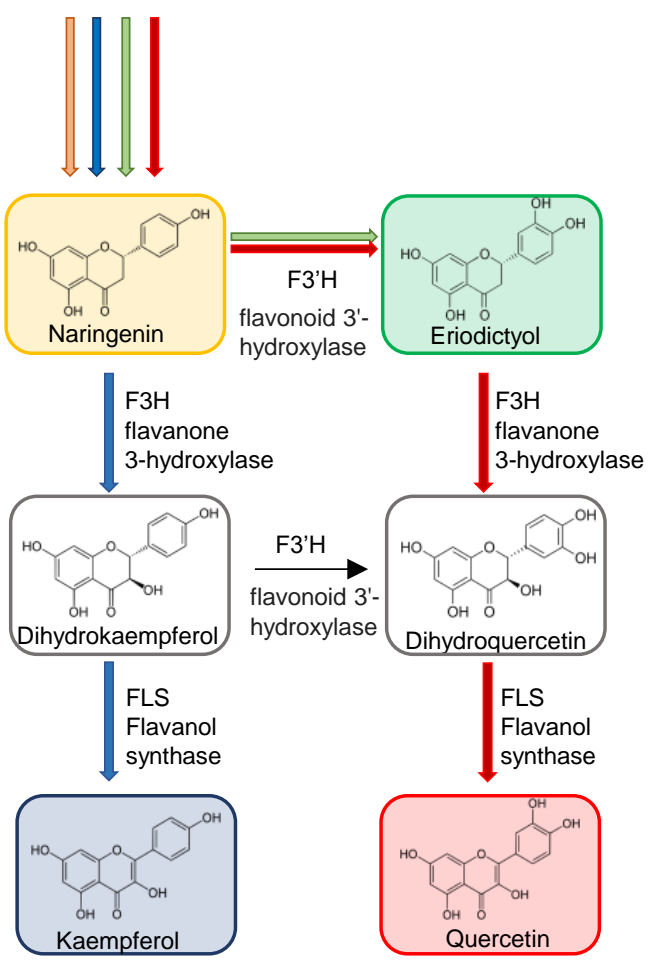

B

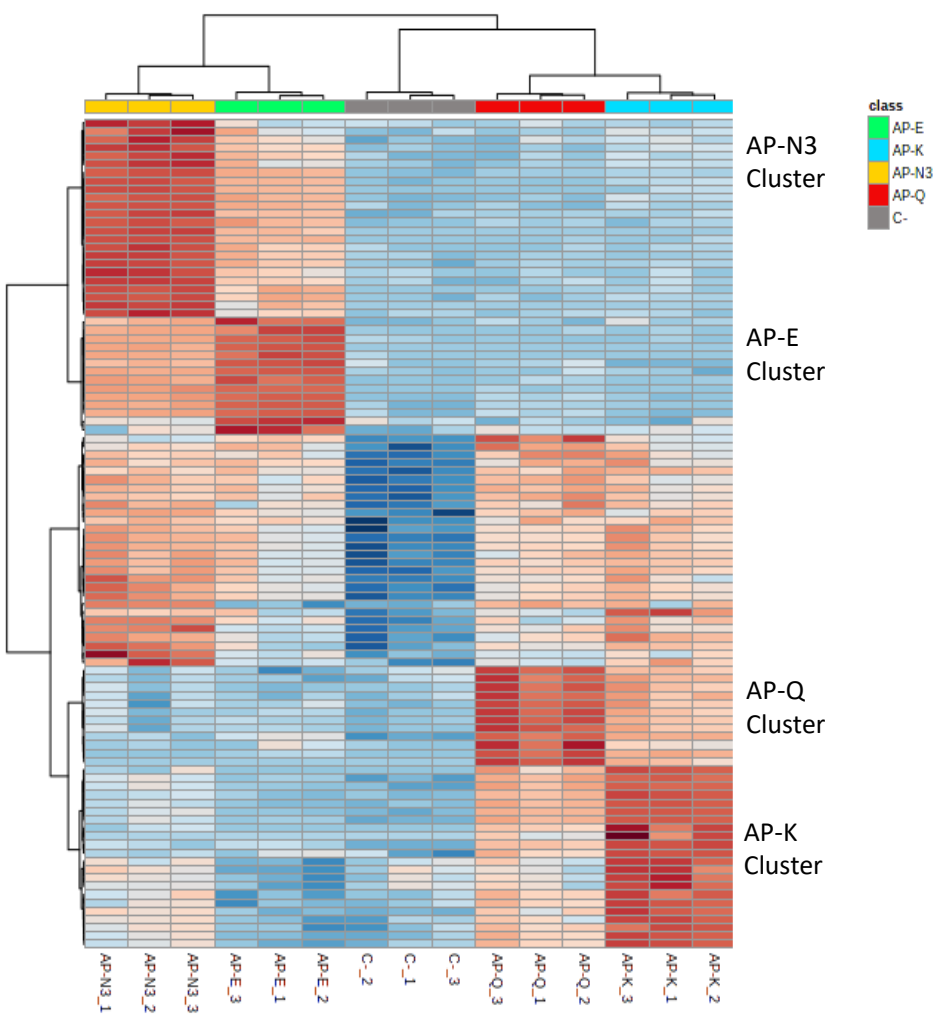

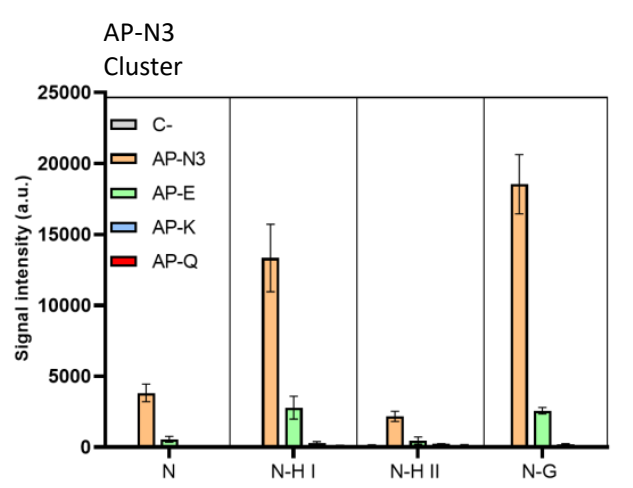

AP-K

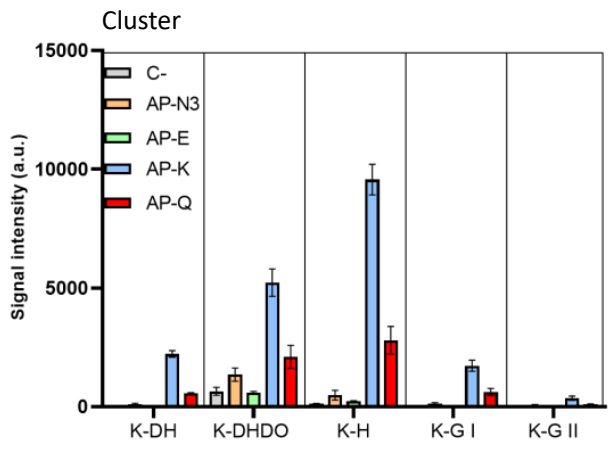

AP-E

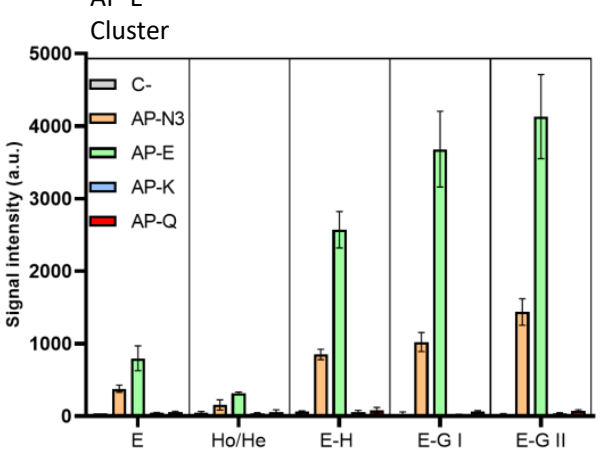

AP-Q

Cluster

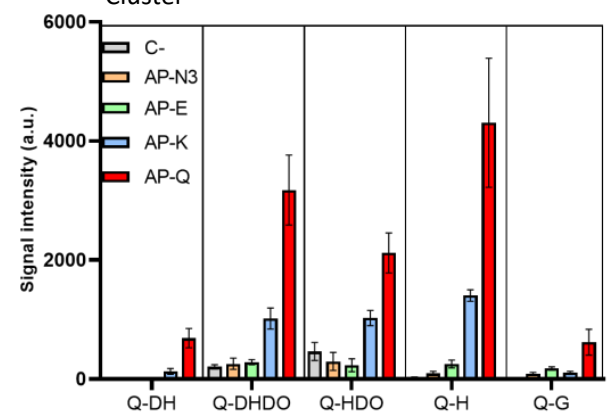

